\title{
A NOTE ON CONTACT STRUCTURES
}

\author{
S. Akbulut and R. Matveyev
}

\begin{abstract}
We construct two contact structures on a homology sphere $M$, that are homotopic through 2-plane fields, but are not isotopic as contact structures. A diffeomorphism of $M$ permutes these two contact structures.
\end{abstract}

Giroux gave examples of homotopic (as 2-plane fields) but not isomorphic tight contact structures on 3-torus ([Gi1], [Gi2]). In [EP] Eliashberg and Polterovich showed that there are homotopic contact structures on $T^{3}$ which are isomorphic but not isotopic (as contact structures). In [LM] Lisca and Matić gave examples of homotopic but not isomorphic tight contact structures on a homology sphere. In this note we give a simple construction of homotopic tight contact structures on a homology sphere which are isomorphic but not isotopic. Unlike the examples of Lisca and Matić, in our example the group of diffeomorphism of the 3-manifold acts nontrivially on the set of path components of oriented contact structures of the manifold, while the action of this group is trivial on the set of path components of the oriented tangent plane distributions. Our construction of contact structures uses theorem of Y. Eliashberg on characterizing compact Stein 4-manifolds with pseudo-convex boundary (PC -manifolds).

Theorem $1([\mathbf{E}])$. Let $X=B^{4} \cup(1$-handles $) \cup(2$-handles $)$ be four-dimensional handlebody with one 0-handle and no 3- or 4-handles. Then:

- The standard PC structure on $B^{4}$ can be extended over 1-handles so that manifold $X_{1}=B^{4} \cup$ (1-handles) has pseudo-convex boundary.

- If each 2-handle is attached to $\partial X_{1}$ along a Legendrian knot with framing one less then Thurston-Bennequin framing of this knot, then the symplectic form on $X_{1}$ can be extended over 2-handles to a symplectic form on $X$, which makes $X$ a PC manifold.

We can visualize pseudo-convex handlebodies as follows: Take a 0-handle to be the unit ball $B^{4}$ in $\mathbf{C}^{2}$ with the induced symplectic structure; choose coordinates in $\mathbf{R}^{3} \subset S^{3}=\partial B^{4}$, so that the induced contact structure $\xi_{0}$ on $\mathbf{R}^{3}$ is the kernel of the form $\lambda_{0}=d z+x d y$. We also assume that the centers of the attaching balls of each 1-handle lies on the plane $\{x=$ constant $\}$.

Projection of a Legendrian link on the plane $\{x=0\}$ has intersection only of the type shown on Figure 1 (coming from left handed crossing), no 
vertical tangencies and all minima and maxima in $y$-direction are cusps. Moreover, every projection with these properties is a projection of some Legendrian link. Thurston-Bennequin invariant of a Legendrian knot $\alpha$ can be calculated by:

$$
t b(\alpha)=b b(\alpha)-(\text { number of right cusps })
$$

where $b b(\alpha)$ is the blackboard (yz-plane) framing of the projection of $\alpha$. Any PC-manifold $X$ induces a tight contact structure on the boundary 3manifold $Y=\partial X$, which is isomorphic to the restriction of the dual $K^{*}$ of the canonical line bundle $K \rightarrow X$. Furthermore if $\alpha$ is an oriented Legenderian knot in $Y$ bounding an oriented surface $F$ in $X$, then the "rotation number" is defined to be the relative Chern class $\operatorname{rot}(\alpha, F)=c_{1}\left(K^{*}, v\right)$ of the induced 2-plane bundle $K^{*} \rightarrow F$ with respect to the tangent vector field $v$ of $\alpha$ (i.e. the obstruction to extending $v$ to a section of $K^{*}$ over $F$ ). In case $Y=S^{3}$ clearly $\operatorname{rot}(\alpha, F)$ does not depend on $F$, and it can be calculated by:

$$
\operatorname{rot}(\alpha)=1 / 2[(\text { number of downward cusps })-(\text { number of upward cusps })]
$$

where "downward" and "upward" are calculated in the obvious way (cf. $[\mathrm{G}])$.

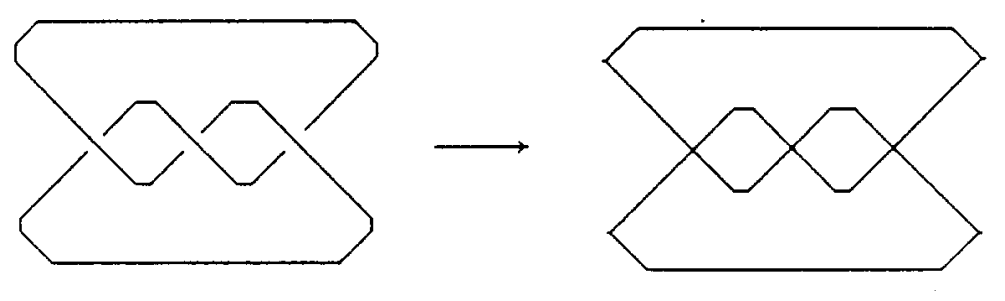

Figure 1.

The proof that the contact structures constructed below are not isotopic rely on the following recent result of P. Kronheimer and T. Mrowka ([KM2]), and P. Lisca and G. Matić ([LM]):

Theorem 2. Let $X$ be a $P C$ manifold, $F \subset X$ be a two-dimensional submanifold of $X$, such that $\alpha=\partial F \subset \partial X$ is Legendrian with respect to induced contact structure and $f$ is framing on $\alpha$ induced by a trivialization of the normal bundle of $F$ in $X$, then $(t b(\alpha)-f)+|\operatorname{rot}(\alpha, F)| \leq-\chi(F)$.

Theorem 2 follows from the adjunction inequality for symplectic manifolds and Theorem 1, and by the assumption that any PC manifold can be 
symplectically imbedded into a closed symplectic manifold with $b_{2}^{+}>1$ (a fact that was proven in $[\mathbf{L M}])$. To show this, attach a 2-handle to $\alpha$ with framing $t b(\alpha)-1$. By Theorem 1 we obtain a $P C$-manifold. Once we are able to symplectically imbed this manifold into a closed symplectic manifold, we can apply the adjunction inequality ([KM2], [MST]) to the closed surface $F^{\prime}=F \cup_{\partial} D$, where $D$ is a 2-disc in the 2-handle, hence:

$$
F^{\prime} \cdot F^{\prime}+\left|K \cdot F^{\prime}\right| \leq-\chi\left(F^{\prime}\right)
$$

where $K$ is canonical class of the closed symplectic manifold. Plugging in the values $F^{\prime} \cdot F^{\prime}=t b(\alpha)-f-1$ and $K \cdot F^{\prime}=-\operatorname{rot}(\alpha, F)$, and $\chi\left(F^{\prime}\right)=\chi(F)+1$ gives the desired inequality.

Consider 4-manifold $W$ with handlebody decomposition shown in the first picture of Figure 2. According to Theorem 1 it can be equipped with a structure of symplectic manifold with pseudo-convex boundary (PC-structure) as shown as in the second picture of Figure 2. This PC structure on $W$ induces a contact structure $\xi$ on 3-manifold $M=\partial W$. Another contact structure $\eta$ on $M$ can be obtained by pulling back $\xi$ via diffeomorphism $f: M \rightarrow M$, where $f$ is the involution on $M$ induced by an involution of $S^{3}$ (as described in [A]) with $f(\gamma)=\gamma^{\prime}$, where $\gamma$ and $\gamma^{\prime}$ are circles in $M$ shown on Figure 2. Thus, we have a contactomorphism $f:(M, \eta) \rightarrow(M, \xi)$, and if $L$ is $\xi$-Legendrian knot in $M$, then $f^{-1}(L)$ is $\eta$-Legendrian and $t b_{\eta}\left(f^{-1}(L)\right)=t b_{\xi}(L)$.

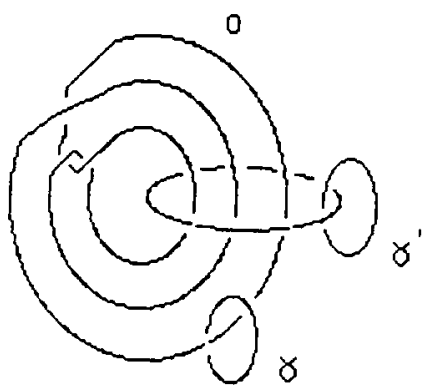

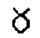

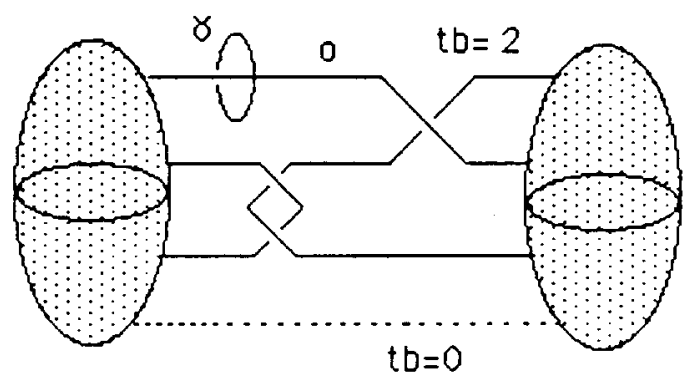

Figure 2.

Proposition. Tight contact structures $\xi$ and $\eta$ are homotopic but not isotopic.

Proof. The fact that $\xi$ and $\eta$ are homotopic is a direct consequence of classification of oriented tangent plane fields on oriented 3-manifolds up to homotopy. Homotopy class of such a field on $M$ corresponds to the homotopy class of a map from $M$ to two-dimensional sphere and, by Thom-Pontryagin construction, to the framed cobordism class of a framed link in $M$. Since 
$M$ is a homology sphere, one can assume that link consist of one unknotted (i.e. bounding an embedded disk) component, and thus homotopy type of the plane field is determined by the framing of this component. This implies that the action of the group of self-diffeomorphisms of $M$ on the set of homotopy types of tangent plane fields is trivial. Therefore, $\xi$ and $\eta$ are homotopic as plane fields.

To prove that $\xi$ and $\eta$ are not isotopic contact structures observe that curve $\gamma$ bounds a smooth embedded disk in $W$. By Theorem 2 we have $t b_{\xi}(K) \leq-1$ for any $\xi$-Legendrian knot $K$ in $M$ isotopic to $\gamma$. On the other hand, isotopy class of knot $\gamma^{\prime}$ has $\xi$-Legendrian representative $L$ with $t b_{\xi}(L)=0$ (dotted loop in Figure 2). Therefore, $K=f^{-1}(L)$ is $\eta$-Legendrian, isotopic to $\gamma$ and $t b_{\eta}(K)=0$. If contact structures $\xi$ and $\eta$ were isotopic, the later equality would contradict inequality $t b_{\xi}(K) \leq-1$.

\section{References}

[A] S. Akbulut, A fake compact contractible 4-manifold, J. Diff. Geom., 33 (1991), 335-356.

[E] Y. Eliashberg, Topological characterization of Stein manifolds of dimension $>2$, International J. of Math., 1(1) (1990), 29-46.

[EP] Y. Eliashberg and L. Polterovich, New applications of Luttinger's surgery, Comment. Math. Helvetici, 65 (1994), 512-522.

[Gi1] E. Giroux, Topologie de contact en dimension 3, Séminaire Bourbaki, 760 (199293), 7-33

[Gi2] Une structure de contact meme tendue, est plus ou moins tordue, Ann. Scient. Éc. Norm. Sup., $4^{e}$ série, 27 (1994), 697-705.

[G] R. Gompf, Handlebody construction of Stein surfaces, preprint.

[KM1] P. Kronheimer and T. Mrowka, On genus of embedded surfaces in the projective plane, Math. Res. Letters, 1 (1994), 797-808.

[KM2] Monopoles and contact structures, to appear.

[LM] P. Lisca and G. Matić, Tight contact structures and Seiberg Witten invariants, preprint.

[MST] J. Morgan, Z. Szabo and C.H. Taubes, The generalized Thom conjecture, to appear.

Received September 17, 1996 and revised October 14, 1996.

Michigan State University

EAST LANSING, MI 48824

E-mail address: akbulut@math.msu.edu

AND

MSRI

BERKELEY, CA 94720

E-mail address: slava@msri.org 